\title{
Histoire, philosophie, iconographie, humanités numériques : une interdisciplinarité difficile
}

\section{Eliana Magnani et Daniel Russo}

\section{(2) OpenEdition \\ 1 Journals}

\section{Édition électronique}

URL : https://journals.openedition.org/cem/12285

DOI : 10.4000/cem.12285

ISSN : 1954-3093

Éditeur

Centre d'études médiévales Saint-Germain d'Auxerre

\section{Référence électronique}

Eliana Magnani et Daniel Russo, « Histoire, philosophie, iconographie, humanités numériques : une interdisciplinarité difficile », Bulletin du centre d'études médiévales d'Auxerre | BUCEMA [En ligne], 16 | 2012, mis en ligne le 05 décembre 2012, consulté le 22 septembre 2022. URL : http:// journals.openedition.org/cem/12285; DOI : https://doi.org/10.4000/cem.12285

\section{Ce document a été généré automatiquement le 22 septembre 2022}

Creative Commons - Attribution - Pas d'Utilisation Commerciale - Partage dans les Mêmes Conditions 4.0 International - CC BY-NC-SA 4.0

https://creativecommons.org/licenses/by-nc-sa/4.0/ 


\title{
Histoire, philosophie, iconographie, humanités numériques : une interdisciplinarité difficile
}

\author{
Eliana Magnani et Daniel Russo
}

1 L'invitation à visiter du point de vue d'historiens médiévistes l'œuvre de Pierre Hadot (1922-2010), à partir de la notion des "exercices spirituels", qui est au centre de sa pensée, nous a conduits à réaliser une série d'opérations, qui, rétrospectivement, donnent matière à réfléchir sur la pratique de l'interdisciplinarité ${ }^{1}$. Une pratique que,

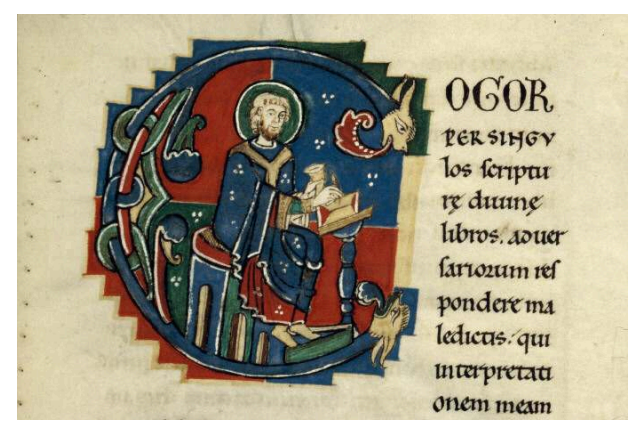
dans nos travaux respectifs, nous cherchons à faire valoir, mais qui ne va pas sans difficultés, tant la prégnance disciplinaire est également à l'œuvre dans cette démarche et dans le dialogue instauré entre disciplines ${ }^{2}$.

2 Nous partons donc de cet exemple concret de recherche, exposons le cheminement adopté et les enseignements à tirer. Après la mise en contexte de l'autonomisation de l'expression des «exercices spirituels" au $\mathrm{xx}^{\mathrm{e}}$ siècle, nous suivons dans la documentation médiévale deux approches complémentaires : celle de l'enquête lexicale menée dans les œuvres d'auteurs ecclésiastiques antérieurs à 1200, en nous servant notamment de l'interrogation informatisée de la Patrologie latine, puis celle de l'analyse de cas dans la "tradition " iconographique du "portrait d'auteur", en prenant en compte les miniatures de la Bible de Saint-Bénigne de Dijon ${ }^{3}$. Ces approches visent à se débarrasser de la convention d'une soi-disant "relation entre les textes et les images ", pour faire porter l'interrogation sur les structures qui doivent être dégagées, depuis les éléments lexicaux et depuis les agencements figuratifs. 
3 En pratiquant ici cet "exercice ", du double côté de l'iconographie et des humanités numériques, dans la perspective d'une démarche historienne, nous voudrions poser la question de l'interdisciplinarité en vis-à-vis d'un parcours philosophique: ses conditions, ses possibilités, ses développements. La notion des « exercices spirituels » étant elle-même ancrée dans le postulat d'une continuité entre l'Antiquité et le Moyen Âge, qui demeure du reste purement analogique, nous examinons de façon critique cette hypothèse et les notions sans cesse mises en avant de "la tradition » et "des influences ».

\section{Contextualiser les « exercices spirituels »}

4 Pierre Hadot, philosophe et historien de la philosophie antique, a occupé la chaire d'histoire de la pensée hellénistique et romaine au Collège de France de 1982 à $1991{ }^{4}$. Son parcours personnel et intellectuel, sur lequel il est revenu en détail dans un livre d'entretiens paru en $2001^{5}$, l'a conduit de la philosophie métaphysique et mystique, à laquelle il s'était consacré pendant ses années de prêtrise (1942-1952), à l'histoire et à la philologie, auxquelles il se forme et pratique ensuite, dans le cadre de différents postes occupés au CNRS (1949-1964), puis en tant que directeur d'études à l'École pratique des hautes études ( $V^{\mathrm{e}}$ section, patristique latine). Ses travaux d'éditeur, de traducteur et de commentateur d'auteurs antiques et tardo-antiques - Marius Victorinus (300 ?-382 ?) ${ }^{6}$, Plotin (205-270) ${ }^{7}$, Ambroise de Milan (340 ?-397) ${ }^{8}$, Marc Aurèle (121-180) ${ }^{9}$, entre autres - nourrissent sa réflexion sur le mouvement de la pensée antique, de la philosophie gréco-romaine au christianisme, du $\mathrm{IV}^{\mathrm{e}}$ siècle av. J.-C. au vi siècle apr. J.-C., réflexion dont il fait la synthèse en 1995 dans Qu'est-ce que la philosophie antique ? ${ }^{10}$.

Selon Pierre Hadot, la philosophie antique n'est pas un discours théorique mais un "mode de vie", où discours et pratique ne sont pas dissociés. "Absorbé » par le christianisme, en passant par le monachisme tardo-antique, ce « modèle » aurait quand même subsisté ensuite jusqu'à nos jours, malgré la rupture que Pierre Hadot attribue à la scolastique, au XIII e siècle, qui aurait signé le "divorce » entre le mode de vie et le discours philosophique. La notion qui sert de fil conducteur et de démonstration à ces postulats est la notion d'« exercices spirituels ", explicitée dans un article publié en 1977 ("Exercices spirituels»), repris dans le recueil de textes paru en 1981 (Exercices spirituels et philosophie antique) ${ }^{11}$. Hadot développe et amplifie alors une idée avancée par Paul Rabbow, dans un ouvrage publié en $1954^{12}$, qui utilisait l'intitulé du célèbre libellum d'Ignace de Loyola (1491-1556), Exercitia spiritualia (1548) ${ }^{13}$, pour désigner, à l'époque impériale, un "système pédagogique ", une "technologie " pour contrôler la vie à travers une éthique pratique. Pierre Hadot considère, à son tour, les Exercitia spiritualia d'Ignace de Loyola, comme «(...) une version chrétienne d'une tradition gréco-romaine (...) », dont il entend « (...) montrer l'ampleur », car, d'après lui, « (...) la notion et le terme d'exercitium spirituale sont attestés dans l'ancien christianisme latin et ils correspondent à l'askesis du christianisme grec (...)». Et cette askesis, il faut l'entendre « (...) non pas comme ascétisme, mais comme pratique d'exercices spirituels (...) ${ }^{14}$. Il analyse alors l'askesis à travers quatre apprentissages, à vivre, à dialoguer, à mourir et à lire. Dans le premier, le ressort essentiel est celui de la conversion qui « (...) bouleverse toute la vie, qui change l'être de celui qui l'accomplit (...) $)^{15}$ : la philosophie est donc, «(...) en premier lieu, une thérapeutique des passions (...)», liée à la « (...) transformation profonde de la manière de voir et d'être de l'individu. Les exercices 
spirituels auront précisément pour objet la réalisation de cette transformation ${ }^{16}$.» Est opéré un changement total de vision sur les choses, qui, de la "vision humaine " passe à une « vision naturelle " pour préciser comment chaque « événement » survient selon une perspective universelle, celle de la "nature universelle » : car, si la philosophie est un « exercice spirituel », la physique l'est tout autant ${ }^{17}$.

6 En revenant plus tard, mais sans changer de point de vue, sur ce qu'il entendait comme le moment de rupture de la philosophie comme mode de vie, Pierre Hadot explique, à l'aide du livre de Juliusz Domański (1927-) qu'il préface ${ }^{18}$, que c'est dans le cadre des universités médiévales, où s'est développée la scolastique au XIII ${ }^{e}$ siècle, que s'opère la "théorisation » de la philosophie comprise désormais comme un discours séparé d'un mode de vie. La « philosophie devient la servante de la théologie ", aidant simplement à "élucider des problèmes théologiques", alors que la théologie assume le rôle de "sagesse supérieure ${ }^{19}$. Hadot nuance, cependant, ses propos antérieurs en pointant que la redécouverte de la philosophie comme mode de vie s'esquisse elle aussi dans les universités médiévales. On comprend très vite, toutefois, que l'enjeu pour lui se situe ailleurs - il s'agit d'une question morale - et que, à travers les universités médiévales, il s'agit aussi de critiquer l'enseignement de la philosophie "théorique » dans les universités contemporaines, conçue pour former «au métier de clerc ou de professeur ", à l'inverse de la "philosophie comme mode de vie", qui vise à former " au métier d'homme" à travers les exercices spirituels, c'est-à-dire à travers le «travail de soi sur soi » ${ }^{20}$.

7 L'emprunt par Pierre Hadot de la notion d'« exercices spirituels » doit être compris dans le contexte de la double opération intellectuelle dont elle a été l'objet, de l'abstraction de l'expression ignacienne et de son historicisation rétrospective.

8 En projetant les Exercices spirituels d'Ignace de Loyola sur la philosophie antique, Paul Rabbow avait lancé l'idée d'une unité des pratiques méditatives païennes et chrétiennes, et créé ainsi, par analogie, une abstraction pour lire les textes d'Épicure, de Sénèque, de Plutarque, d'Épictète et de Marc Aurèle ${ }^{21}$. Cette projection/abstraction, qui semble alors nouvelle, a été reçue différemment selon qu'on ait accepté ou non son opérabilité. D'un côté, certains auteurs trouvent l'analogie injustifiée, considérant qu'aucune évidence dans les textes permet de postuler une connexion directe entre les pratiques anciennes et chrétiennes ${ }^{22}$; d'un autre côté, cette analogie sera élevée comme un nouveau paradigme qu'il sera question de perfectionner, d'amplifier. Pierre Hadot, à la suite de son épouse, Ilsetraut Hadot ${ }^{23}$, va emprunter cette voie en proposant dans le deuxième chapitre du recueil d'articles de 1981, «Exercices spirituels antiques et "philosophie chrétienne" ", la construction d'une continuité entre la philosophie antique et le christianisme - ou l'" absorption de la philosophia par le christianisme »-, en passant par les mouvements monastiques des premiers siècles du christianisme ${ }^{24}$.

9 En développant la théorie de Rabbow, Pierre Hadot, élevé «dans les jupes de l'Église » ${ }^{25}$, n'ignorait pas le mouvement contemporain de la constitution progressive d'une théologie spirituelle - ou théologie mystique, ou théologie ascétique - comme discipline au sein des institutions romaines, ce qui avait conduit à la création de l'Istituto di spiritualità à l'intérieur de la Pontificia università gregoriana en $19588^{26}$, et s'était traduit, entre autres, par l'historicisation rétrospective et l'autonomisation des « exercices spirituels» de Loyola. L'un des premiers jalons de cette autre opération intellectuelle se retrouve dans un court article du jésuite Ludwig Hertling (1895-1980) ${ }^{27}$, de 1933, contenant le répertoire des auteurs du haut Moyen Âge ayant 
employé, avant de Loyola, l'expression "exercices spirituels", au singulier ou au pluriel, en particulier dans des récits hagiographiques ${ }^{28}$. Selon Hertling, les expressions exercitium spirituale et exercitia spiritualia désignent dans ces textes "les actions qui composent et régissent la vie ascétique ", dans un sens pas très éloigné de la première annotation d'Ignace de Loyola : «(...) quod hoc nomine exercitiorum spiritualium intelligitur quilibet modus examinandi conscientiam, meditandi, contemplandi, orandi vocaliter et mentaliter, et aliarum operationum spiritualium (...) ». De Loyola et ses Exercices sont placés ainsi dans une longue lignée de spirituels et de saints. Un second jalon important est la transformation de l'expression "exercices spirituels " en une formule autonome à travers son emploi, pour la première fois, comme entrée d'un dictionnaire encyclopédique, en l'occurrence dans le tome 4 du Dictionnaire de spiritualité publié en 1961. L'entreprise monumentale du Dictionnaire de spiritualité. Ascétique et mystique. Doctrine et histoire a été conçue en 1928 et a été publiée entre 1932 et 1995, sous la responsabilité de la Compagnie de Jésus. Dans la longue notice rédigée par Jean Leclercq (1911-1993), moine bénédictin, professeur à la Gregoriana, par le jésuite André Rayez, par le rédemptoriste Pierre Debongnie et par Charles Schmerber, la notion d'«exercices spirituels» est inscrite dans l'histoire des écrits et des pratiques chrétiennes, depuis l'Antiquité jusqu'au $\mathrm{xx}^{\mathrm{e}}$ siècle ${ }^{29}$. Jean Leclercq, auteur du chapitre dédié à l'Antiquité et au haut Moyen Âge, rappelle en philologue, les «sens profanes " d'un terme, exercitium, qui ne figure qu'une seule fois dans la Bible, et dont l'acception, celle d'une " action qui exige effort ", dans le domaine de "l'œuvre à faire " et de "la réflexion ", a très tôt revêtu une connotation militaire. C'est d'abord dans cette nuance de "lutte » qu'exercitium aurait été, selon lui, employé dans la patristique chrétienne, pour rendre compte de l'« effort qu'exige la vie spirituelle », de l'ascèse. L'enjeu de l'inscription des "exercices spirituels» dans l'histoire du christianisme, "aux origines ", est ici idéologiquement important, car, aux lendemains de la seconde guerre mondiale et à la veille de Vatican II, il s'agit de confondre les " exercices spirituels » ainsi historicisés avec les "exercices de piété ", qui concernent aussi bien le magistère des prêtres que la place des laïcs dans l'Église. L'introduction de l'article l'énonce clairement : les termes exercitium, exercitia et exercitatio désignent les pratiques de la vie spirituelle depuis les premiers siècles et ont une histoire très homogène, tandis qu'au Moyen Âge les expressions exercitium spirituale, exercitia spiritualia viennent à désigner des « exercices de piété »; c'est à ces exercices de piété, depuis l'encyclique Mediator Dei de Pie XII, de 1947 - «L'ensemble du culte que l'Église rend à Dieu doit être à la fois intérieur et extérieur »--, que les dix dernières pages de la notice sont dédiées.

L'utilisation par Pierre Hadot de la notion d'« exercices spirituels " pour essayer de traduire la pratique philosophique de l'Antiquité (mais pas seulement), n'est donc pas un choix anodin. Elle s'inscrit dans ce double mouvement de surinvestissement des ecclésiastiques, des théologiens et des historiens de la philosophie dans la création d'un universel atemporel pour rendre compte d'attentes morales, tout à fait contemporaines. Du reste, toute une génération de chercheurs est traversée par les mêmes préoccupations qui agitent ce qu'on nommait alors le champ des sciences humaines : en histoire, Lucien Febvre (1878-1956) ${ }^{30}$; en iconographie, André Grabar (1896-1990), lecteur de Ferdinand de Saussure (1857-1913) et de ses observations sur les "faits linguistiques ", dont il s'inspira pour décrire les "faits iconographiques " ${ }^{31}$; en esthétique, Edgar de Bruyne (1898-1959) ${ }^{32}$; en linguistique, Émile Benveniste (1902-1976) ${ }^{33}$; en histoire des arts, Jean Seznec (1905-1983) et Jean Adhémar 
(1908-1987) ${ }^{34}$; en histoire de la littérature antique, Pierre Courcelle (1912-1980), auquel Pierre Hadot dédiait précisément son livre de $1981^{35}$.

11 Au titre d'une simple incise, nous mesurons ici l'étendue du contraste entre l'approche de Pierre Hadot et la recherche philosophique, qui, aujourd'hui, considère le monachisme tardo-antique et médiéval comme une expérience en soi, propre à faire émerger de nouveaux concepts. Nous pensons en particulier au concept «forme-devie », que Giorgio Agamben (1942-) a développé en interrogeant la relation qui peut s'établir entre la règle et la vie monastique (regula et vita), et qui rend compte d'une "zone d'indifférenciation" aussi entre règle et forme de vie, c'est-à-dire, d'une communauté en deçà du droit, dont la structure serait consubstantielle à ses normes plutôt que mise en forme par elles - ce ne serait pas la règle qui créerait la forme de vie, mais plutôt la forme de vie qui créerait la règle; la transformation de toute la vie en un office divin (liturgie) étant l'expansion maximale de la normativité monastique aux moindres détails de la vie ${ }^{36}$.

\section{Tester les « exercices spirituels ». Les mots}

Une fois précisé le contexte dans lequel la notion d'« exercices spirituels » émerge comme une formule autonome, il reste à penser dans quelle mesure elle aurait été effectivement opératoire pour l'historien médiéviste, qui dispose aujourd'hui d'outils et de méthodes de recherche différents de ceux utilisés par les auteurs du Dictionnaire de spiritualité ou par Pierre Hadot. La masse des textes médiévaux numérisés interrogés à l'aide de logiciels réalisant la recherche de cooccurrences, l'affichage par mot-clé, par distribution chronologique et par fréquence, autorise la lecture de milliers de documents en déplaçant l'attention du texte vers les différentes échelles, plus petites ou plus grandes, des unités lexicales ${ }^{37}$.

Après avoir effectué la recherche inversée dans les dictionnaires électroniques du latin médiéval ${ }^{38}$ et dans la Vulgate ${ }^{39}$, nous avons réalisé l'expérience autour du terme latin exercitium dans l'ensemble des volumes de la Patrologie latine ${ }^{40}$, interrogée avec le logiciel PhiloLogic ${ }^{41}$. Il s'agissait d'abord de repérer les principales cooccurrences de la troncature exerciti*, ensuite de vérifier la répartition chronologique des deux cooccurrences exerciti* ${ }^{*}$ vertu* et exerciti $^{*}+$ spiritual $^{*}$, enfin celle de l'expression ignacienne exercitia spiritualia. Outre les mots de la pratique et ceux des valeurs monastiques, ces recherches préliminaires pointent surtout vers le XII ${ }^{e}$ siècle.

Réunissant environ 5645 documents latins d'auteurs chrétiens antérieurs à 1200, de Tertullien (ca. 150/160-ca. 220) à Innocent III (1160-1216), la Patrologie contient environ 105 millions de mots. L'interrogation de la troncature exerciti* ne donne que 3291 occurrences réparties dans 949 œuvres ${ }^{42}$. 
Tab. 1 - Principales cooccurrences de exerciti*.

\begin{tabular}{|c|c|c|}
\hline 1. & virtus & 357 \\
\hline 2. & opus & 283 \\
\hline 3. & bonum & 249 \\
\hline 4. & spiritalis & 238 \\
\hline 5. & labor & 158 \\
\hline 6. & studium & 124 \\
\hline 7. & vita & 112 \\
\hline 8. & alius & 99 \\
\hline 9. & omnis & 95 \\
\hline 10. & corpus & 86 \\
\hline 11. & ille & 68 \\
\hline 12. & suus & 67 \\
\hline 13. & habeo & 55 \\
\hline 14. & semper & 54 \\
\hline 15. & usus & 53 \\
\hline 16. & seu/sive & 49 \\
\hline 17. & religio & 47 \\
\hline 18. & sancta & 46 \\
\hline 19. & itaque & 44 \\
\hline 20. & mens & 44 \\
\hline 21. & missus & 44 \\
\hline 22. & tam & 44 \\
\hline
\end{tabular}

$\begin{array}{lll}\text { 23. hic } & 42 \\ \text { 24. } & \text { pietas } & 40 \\ \text { 25. disciplina } & 39 \\ \text { 26. } \text { rex } & 39 \\ \text { 27. humilitas } & 38 \\ \text { 28. magis } & 34 \\ \text { 29. } & \text { actio } & 33 \\ \text { 30. qui } & 33 \\ \text { 31. longus } & 31 \\ \text { 32. } \text { scilicet } & 31 \\ \text { 33. conversatio } & 30 \\ \text { 34. quatuor } & 29 \\ \text { 35. apud } & 27 \\ \text { 36. homicida } & 27 \\ \text { 37. hujusmodi } & 27 \\ \text { 38. militia } & 27 \\ \text { 39. namque } & 26 \\ \text { 40. noster } & 26 \\ \text { 41. perdo } & 26 \\ \text { 42. videlicet } & 26 \\ \text { 43. circa } & 25 \\ \text { 44. lectio } & 24\end{array}$

\begin{tabular}{|c|c|}
\hline 45. & divinus \\
\hline 46. & fides \\
\hline 47. & facio \\
\hline 48. & caput \\
\hline 49. & coelestis \\
\hline 50. & nullus \\
\hline 51. & quando \\
\hline 52. & vitium \\
\hline 53. & cap \\
\hline 54. & contemplatio \\
\hline 55. & justitia \\
\hline 56. & juxta \\
\hline 57. & tantus \\
\hline 58. & caro \\
\hline 59. & cor \\
\hline 60. & sum \\
\hline 61. & manus \\
\hline 62. & modo \\
\hline 63. & natura \\
\hline 64. & quidam \\
\hline 65. & solus \\
\hline 66. & tempus \\
\hline
\end{tabular}

Le tableau 1 des lemmes des principales cooccurrences, dans un rayon de cinq mots avant et après exerciti*, indique qu'il est le plus souvent associé à virtus, ensuite à bonum opus, et à spiritalis. Suivent une série de termes qui composent, avec les précédents, le champ sémantique de la vie consacrée à Dieu - labor; studium; vita ; religio ; pietas ; disciplina; humilitas ; conversatio ; lectio ; coelestis ; contemplatio ; justitia -, de même que des antonymes qui renvoient au passage (conversatio) d'un terme à l'autre de pôles opposés: vitium/virtus; caro-corpus/spiritalis. Une autre série de lemmes relève du contexte formulaire, c'est-à-dire les mots cooccurrents qui proviennent d'un passage de la Bible. Missus, rex, homicida, perdo font partie de Matthieu 22, 7 dans la parabole du festin de noces ${ }^{43}$. La cooccurrence militia se situe dans ce champ sémantique martial d'exercitium, champ qui devient central dans le monachisme médiéval ${ }^{44}$. Corpus et pietas peuvent, quant à eux, renvoyer à 1 Timothée 4, 7-8 ${ }^{45}$, passage paulinien qui servira de fondement à la distinction entre exercices corporels et exercices de piété ${ }^{46}$, distinction qui ouvre à la déclinaison épithétique d'exercitium.

D'un point de vue chronologique, l'essai de répartition de deux cooccurrences, exerciti* + vertu* - la plus fréquente dans les textes de la Patrologie latine - et exerciti + spiritual*, indique que les deux cooccurrences sont actives aux mêmes moments : au IV $\mathrm{IV}^{\mathrm{e}}, \mathrm{au} \mathrm{Ix}^{\mathrm{e}}$, au $\mathrm{XI}^{\mathrm{e}}$ et surtout au XII ${ }^{\mathrm{e}}$ siècle (tab. 2 et graph. 1). 
Tab. 2 - Cooccurrences par siècle.

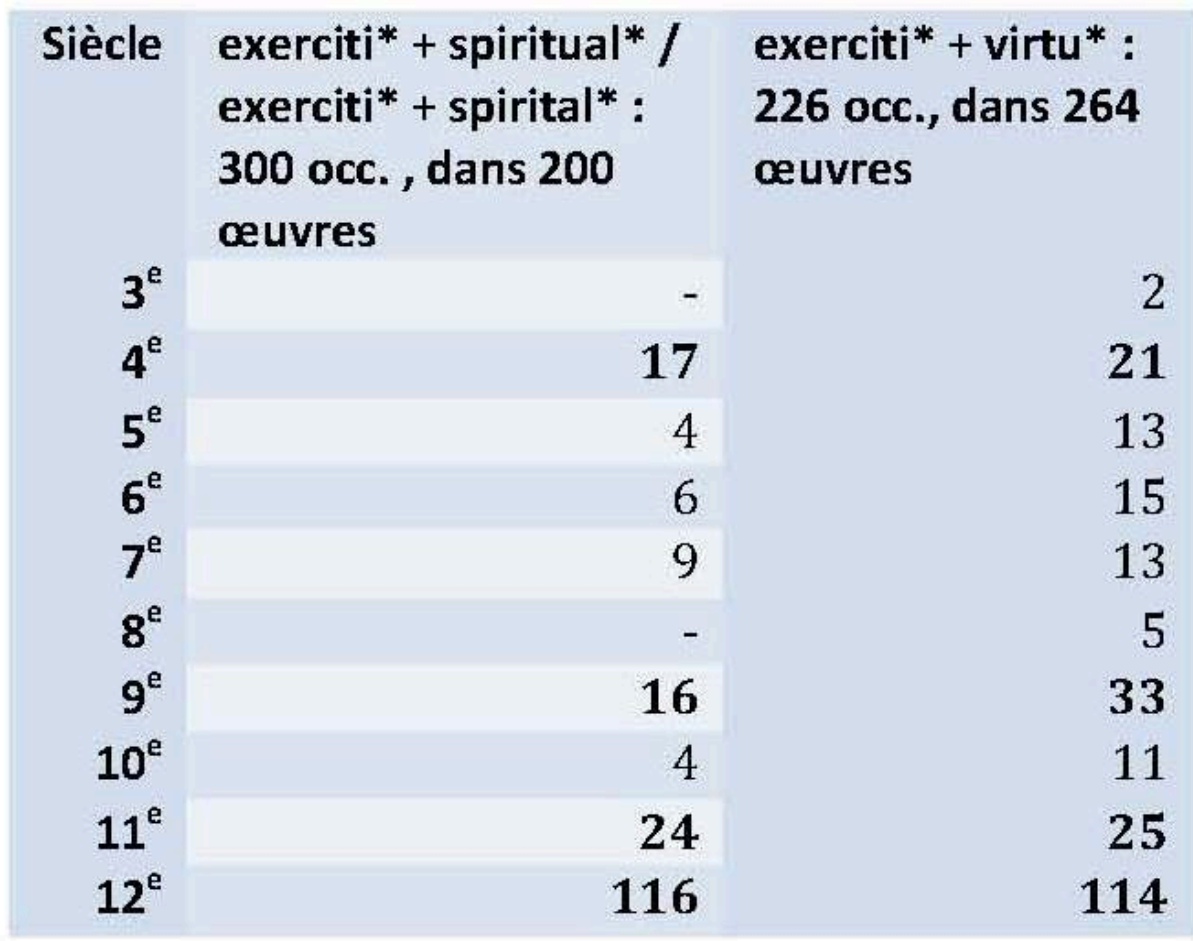

Graph. 1 - Cooccurrences par siècle.

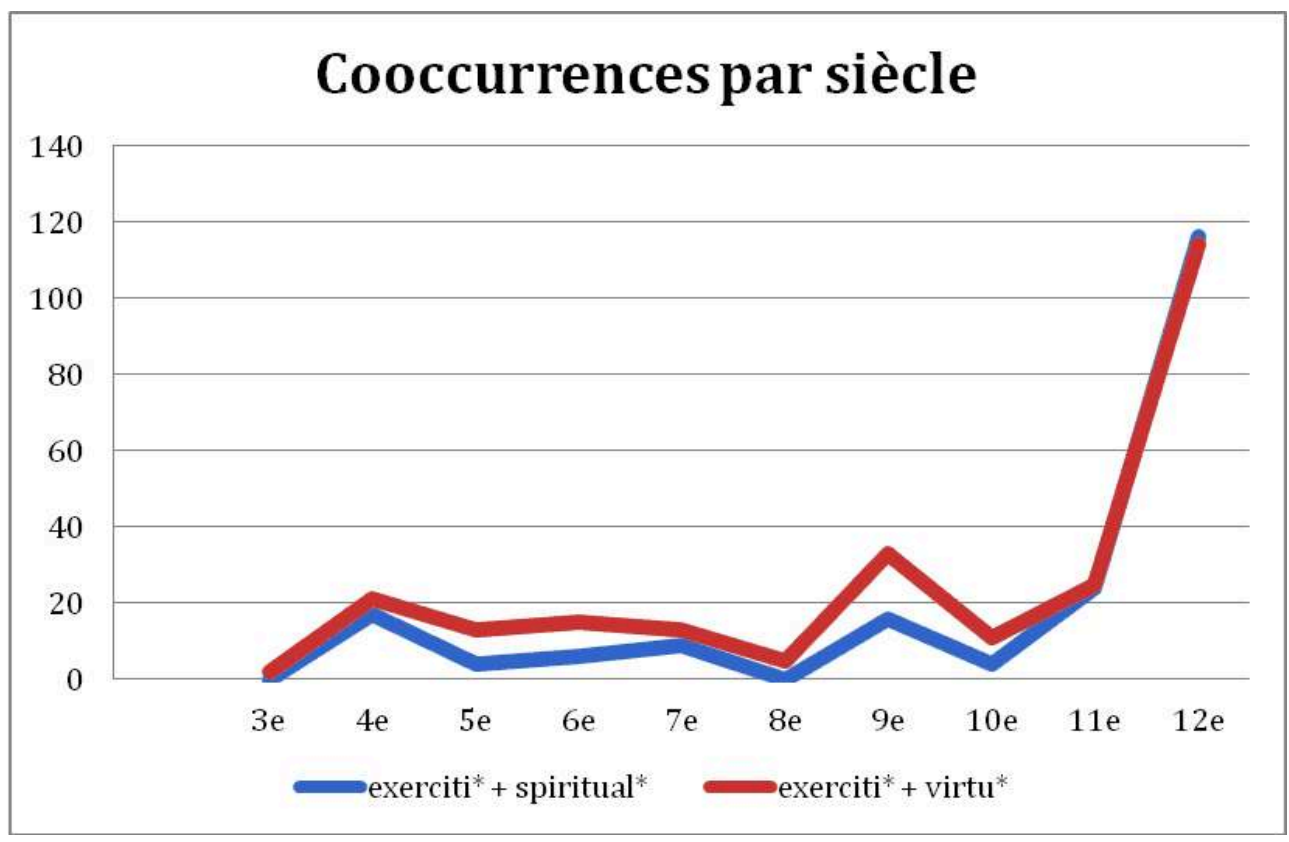

17 Or, tous ces moments coïncident avec des mouvements monastiques importants. Le $\mathrm{IV}^{\mathrm{e}}$ siècle est la période d'épanouissement du premier monachisme, sur lequel se penchent les Pères grecs et latins. Le $\mathrm{IX}^{\mathrm{e}}$ siècle est l'époque de la réforme monastique carolingienne, qui entend uniformiser le mode de vie des communautés par l'imposition de la règle bénédictine. Les $\mathrm{XI}^{\mathrm{e}}$ et $\mathrm{XII}^{\mathrm{e}}$ sont les siècles des débuts puis du développement de la réforme ecclésiastique dite "grégorienne» et du «nouveau 
monachisme" imprégné d'un fort ascétisme, dont l'enjeu sociétal était de bien distinguer les sphères ecclésiastiques et laïques.

L'enquête dans la Patrologie latine sur le syntagme précis exercitia spiritualia, qui a été utilisé par Ignace de Loyola pour nommer son libellum, confirme pour l'emploi de ces termes la centralité du XII ${ }^{\mathrm{e}}$ siècle. L'expression n'apparaît que 25 fois, mais 23 mentions datent du XII ${ }^{\mathrm{e}}$ siècle. À part un anonyme du $\mathrm{VI}^{\mathrm{e}}$ siècle ${ }^{47}$ et Dunstan de Cantorbéry (ca. 909-980), abbé et archevêque $d u \mathrm{x}^{\mathrm{e}}$ siècle ${ }^{48}$, les autres mentions proviennent surtout des auteurs issus du nouveau monachisme : les cisterciens Bernard de Clairvaux (ca. 1090-1153) ${ }^{49}$, Aelred de Rielvaux (1110-1166/1167) ${ }^{50}$ et Arnoul de Bohéries ( $\dagger$ ca. 1140) ? $^{51}$; le chartreux Bernard de Portes ( $\dagger$ ca. 1156/1159) ${ }^{52}$; le victorin Richard de Saint-Victor (ca. 1110-1173) ${ }^{53}$; le bénédictin réformateur Guillaume de Saint-Thierry (ca. 1085-1148) ${ }^{54}$; le poète et théologien Pierre de Blois (ca. 1130/1135-1211/1212) ${ }^{55}$; en plus de deux ouvrages anonymes du XII ${ }^{\mathrm{e}}$ siècle ${ }^{56}$. Le lexicogramme (graph. 2), issu de l'ensemble des paragraphes contenant l'expression, permet de visualiser les mots exercitia et spiritualia conjugués avec un troisième terme, corporalia ${ }^{57}$.

Graph. 2 - Lexicogramme. Exercitia spiritualia dans la Patrologie latine.

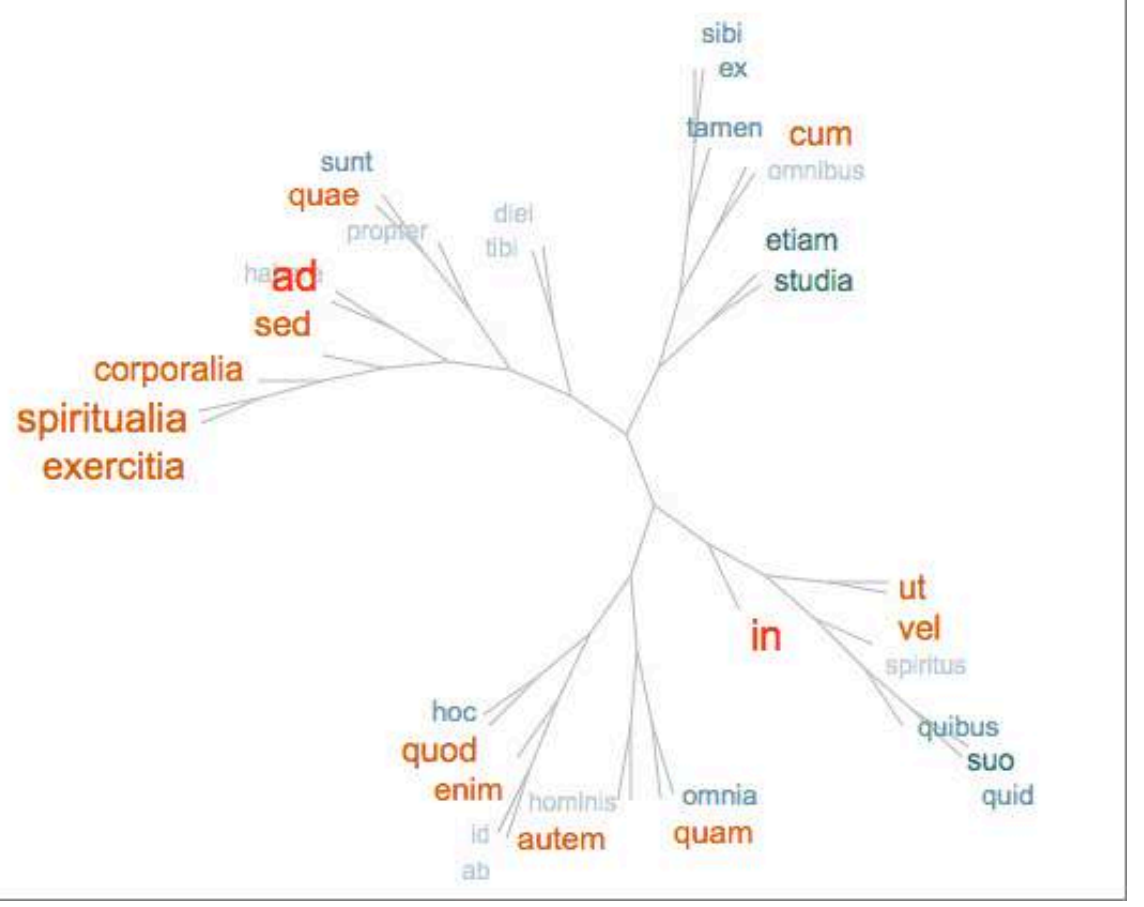

19 Outre l'écho avec la première épître à Timothée 4, 7-8, l'opposition chair/esprit renvoie dans le contexte monastique non seulement au combat premier de la vie monastique, mais aussi aux vifs débats qui jalonnent la période sur la nature du Christ et le réalisme eucharistique, sa présence en chair lors de la communion. La recrudescence même de l'emploi d'exercitia amène à restituer, par ailleurs, son acception militaire comme référence propre au monachisme : les moines se pensent en tant que guerriers, milites Christi, et cette identité, dans une société rendue de plus en plus martiale et référée au divin, se construit dans l'échange constant avec les laïcs porteurs d'armes. 
20 Par rapport aux « exercices spirituels » tels que les pose Pierre Hadot, de la continuité chrétienne de la pratique philosophique tardo-antique à la rupture entre theologia et philosophia qu'il localise au XIII ${ }^{\mathrm{e}}$ siècle, au moment de la scolastique, ou par rapport à la construction vectorisée de l'Antiquité chrétienne jusqu'au $\mathrm{xx}^{\mathrm{e}}$ siècle, dans la notice « exercices spirituels » du Dictionnaire de spiritualité, la recherche préliminaire dans une masse considérable de textes, qu'il faudra approfondir et élargir au-delà de 1200, soulève plusieurs questions. Pour commencer, étant donné la très faible fréquence d'occurrences, il semble difficile de soutenir, historiquement, une centralité de l'expression «exercices spirituels» avant son élaboration ignacienne. L'enquête préliminaire ayant pointé le $\mathrm{XII}^{\mathrm{e}}$ siècle et le nouveau monachisme, le passage tardoantique doit être repensé. Il faudra aussi considérer la concomitance entre les débuts de la scolastique et la première cristallisation des cooccurrences de exerciti* ${ }^{*}$ spiritual $^{*}$ et de exerciti* + vertu* dans les milieux monastiques, et nous interroger sur le contenu de leurs prolongements possibles au XIII ${ }^{\mathrm{e}}$ siècle, et au-delà, dans des courants spirituels et mystiques - des ordres mendiants aux confréries laïques, en passant par les béguinages, par exemple.

\section{Tester les « exercices spirituels ». La « tradition » iconographique du " portrait d'auteur»}

21 Avant même que l'enquête sur les mots dans la Patrologie latine ait circonscrit le $\mathrm{XII}^{\mathrm{e}}$ siècle et le nouveau monachisme, comme le moment chronologique d'une première cristallisation de l'association entre le substantif exercitia et l'adjectif spiritualia (mais aussi corporalia), nous avions pensé que la notion des « exercices spirituels » de Pierre Hadot, d'un point de vue des ressources iconographiques, serait à confronter avec les productions monastiques du $\mathrm{XII}^{\mathrm{e}}$ siècle, afin de critiquer les idées passe-partout de «tradition » et d'« influence » antique. Car c'est alors qu'émerge une nouveauté, celle de la figuration du champ, campus, du fond de l'image, qui nous semblait obliger à reconsidérer, voire dépasser, la catégorie dès lors problématique de la continuité du " portrait d'auteur " gréco-romain. Au même moment, en effet, le champ, campus, peint à l'intérieur du livre, puis dans l'initiale figurée, devient d'un emploi assez courant pour désigner le «fond de l'image », en étroite association avec l'instance qui institue ces commandes et ces réalisations d'envergure, l'Église et tout son appareil de discours. À l'issue d'une analyse trop brève et qui se termine maladroitement dans le symbolisme, Jean Wirth a pointé l'attention vers cette articulation neuve ${ }^{58}$. À partir d'une autre position, mais encore avec une formation très différente de celle de Pierre Hadot, comme nous l'avons noté précédemment, des philosophes se sont montrés intéressés par ce problème de figuration, celui de l'implicite d'une présence, parfois en relation d'essence avec la reformulation monastique des formes de vie et le développement d'une ontologie autre qui traverse l'imaginaire et son « champ ${ }^{59}$.

Pierre Hadot est, certes, à situer selon de tout autres perspectives, les «exercices spirituels » conduisant à la transformation de l'être du sujet qui s'exerce "spirituellement" sur lui-même ${ }^{60}$. Plusieurs traits circonscrivent les étapes de la transformation. Certains de ces traits relèvent de l'attention, et sont du côté de la méditation et du souvenir de ce qui est; d'autres, de l'intellect, et soulignent la part importante prise par la lecture, l'audition, la recherche, l'examen approfondi de soi, au cours de tout le processus décrit qui n'est autre que celui-là même de la vie psychique ; 
d'autres encore, de l'action sur soi mettant en valeur la maîtrise de soi, l'accomplissement des devoirs, l'indifférence aux choses indifférentes, dans le vocabulaire de Sénèque ( 4 av. J.-C.-65 apr. J.-C..) ; d'autres enfin, des exercices pratiques qui étaient destinés à susciter des habitudes. Pierre Hadot fait surgir des catégories qui explicitent tout le pan de l'imaginaire saisi, analysé, sous l'angle de la phénoménologie de la vie psychique et, en bien des points de son analyse, il répond à des questions posées par Jean-Paul Sartre, surtout dans L'imaginaire. Psychologie phénoménologique de l'imagination ${ }^{61}$. C'est aussi par là qu'il pourrait être rapproché, dans un rapport analogique toutefois, de la situation médiévale dans ce qu'elle a de plus extérieurement accessible.

Le champ d'expression iconographique choisi s'avère, à cet égard, pertinent pour une première série d'observations à des moments d'affirmation de cette idée de "renaissance» de l'antique et de reformulation de tout le vocabulaire des signes visuels et textuels du fonds monastique d'inspiration carolingienne ${ }^{62}$. Le XII ${ }^{\mathrm{e}}$ siècle fut, par excellence, l'époque de toutes les ruptures dans tous les domaines de la société, y compris dans la manière d'envisager celle-ci comme un ensemble composé de tous ses éléments reliés entre eux et au tout. Dans bien des cas, les moines furent au premier rang des changements. Les monastères devinrent en quelque sorte les laboratoires au centre des nouvelles expériences qui servirent, souvent, de bases aux transformations sociales. Nous citerons l'exemple du livre et, dans le livre monastique, l'exemple de l'image de "l'auteur monastique » en train d'écrire, reprenant et, en même temps, reformulant de fond en comble le champ du "portrait d'auteur » gréco-romain, puis tardo-antique ${ }^{63}$. Sur les miniatures de la Bible de Saint-Bénigne de Dijon, une abbaye possédant sans doute un scriptorium monastique dans la refonte de l'an Mil, sous l'abbatiat de Guillaume de Volpiano (962-1031, abbé de 990 à 1001 ?), et en rapport au $\mathrm{XII}^{\mathrm{e}}$ siècle avec le scriptorium de Cîteaux, saint Jérôme (ca. 347-420) incarna, c'est-à-dire donna le support de son personnage de moine traducteur de la Bible, aux formes et aux figures de l'«auteur » monastique montré en plein exercice de son labeur qui serait, aussi, un « exercice spirituel », dans la «tradition » de ces autres exercices qu'étudiait Pierre Hadot ${ }^{64}$. Autour du deuxième quart du XII siècle, sur les feuillets de la Bible composée pour une grande abbaye, le moine Jérôme appliqué à traduire les textes saints de l'hébreu au latin signe le " portrait de l'auteur » inspiré par le Verbe de Dieu et participant à la construction de l'Église.

Voyons quelques exemples de ces procédés sur les fonds des quatre initiales peintes et montrant dans la Bible choisie tout le travail effectué par le(s) scribe(s) sur le « champ ». Au feuillet $6 \mathrm{r}^{\circ}$, au cœur de la lettrine $D$ (fig. 1), Jérôme est figuré tout à la fois comme l'objet et le sujet de l'enluminure, le destinataire de l'échange de lettre avec Desiderius et l'autorité à laquelle est soumise une question de traduction ${ }^{65}$. 
Fig. 1 - Bible de Saint-Bénigne de Dijon, DIJoN, Bibliothèque municipale, ms. 2, fol. $6 \mathrm{r}^{\circ}$, initiale $D, 2^{\mathrm{e}}$ quart du XII siècle? (source : enluminures.culture.fr).

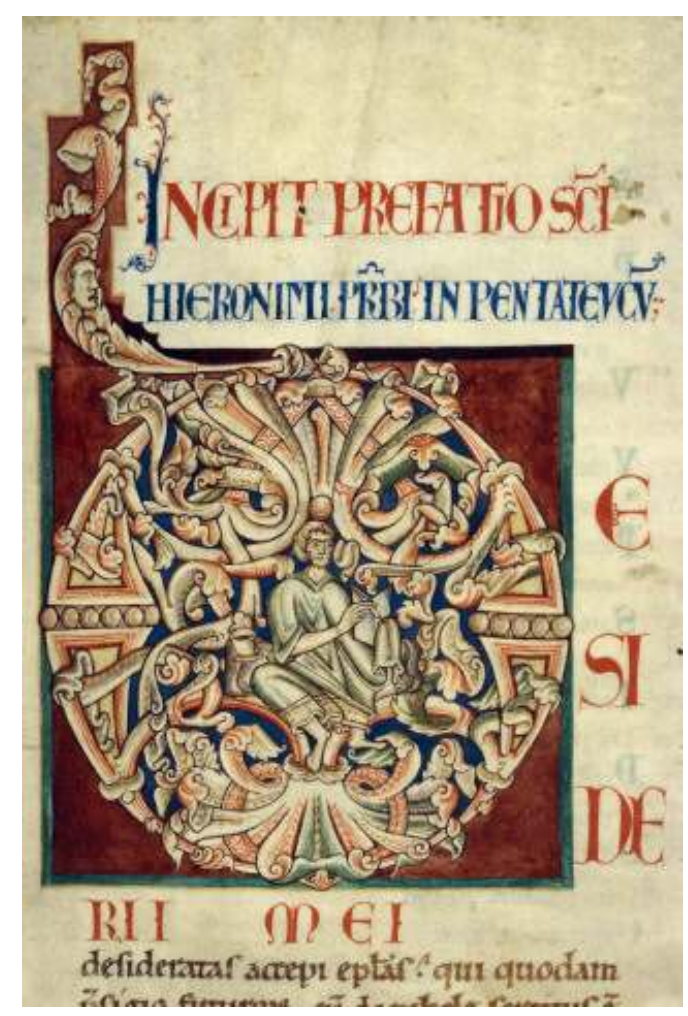

Il émerge du champ comme une figure, autrement dit comme une forme imaginée à partir de l'entrelacs du végétal qui la configure, tant à partir de la jonction des deux feuilles d'acanthe ouvertes, disposées en un mouvement inversé l'une par rapport à l'autre, qu'à partir aussi de leurs ramifications et de leurs boutons : entre autres, le(s) scribe(s) insiste(nt) sur l'emplacement de la tête de saint Jérôme par la peinture d'un bouton végétal terminant la feuille d'acanthe du haut, juste au-dessus de sa tête, tout auprès de la tonsure, la soulignant davantage. Plus avant, au feuillet $100{ }^{\circ}$, Jérôme est assis à l'intérieur du cartouche qui forme le montant de la lettre initiale $U$ (fig. 2) : il a suspendu son écriture, il a la main droite posée sur la joue et, de l'autre main, il tient une plume d'oie posée à plat sur le bi-feuillet doré du codex sur ses genoux. 
Fig. 2 - Bible de Saint-Bénigne de Dijon, op. cit., fol. 100rº, initiale $U$.

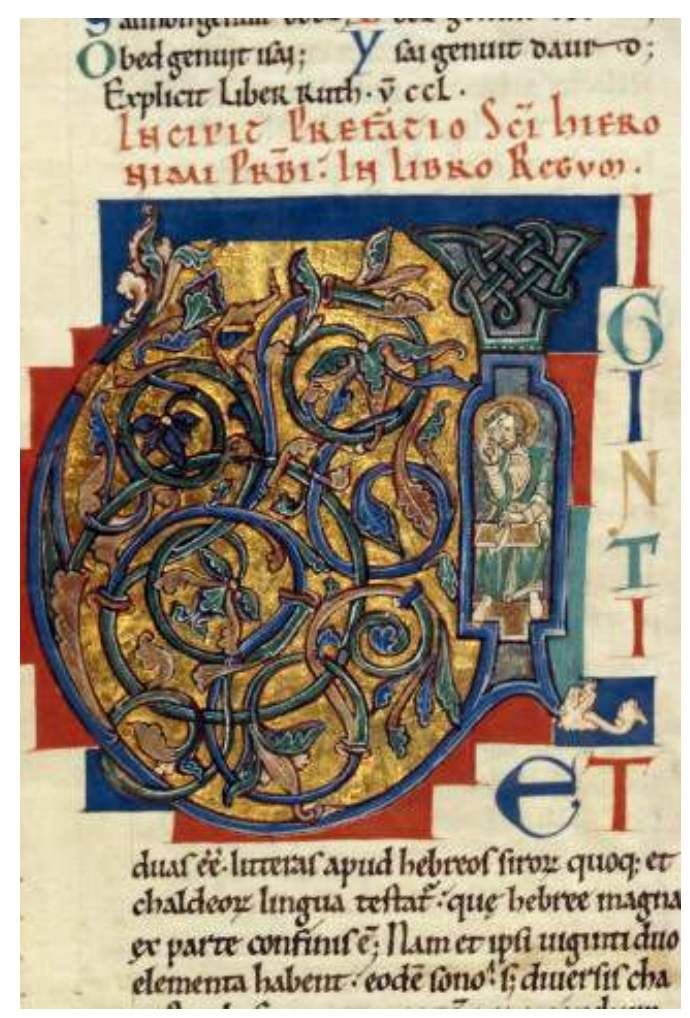

Nimbé, l'auteur monastique est contenu dans son support, la lettrine qui lui sert d'attribut-présentoir et le dévoile au cours d'un instant de méditation, d'observation également, sur ce qui se passe à l'intérieur de l'initiale: un mélange de tiges et de feuilles, s'achevant parfois en fleurs écloses et paraissant l'image de ce qui est tout agitation dans le corps même de l'initiale. Le fond est peint à la feuille d'or ${ }^{6}$. Tout l'intérieur supporte des feuilles et des fleurs en volutes et en rinceaux qui ont été déposées en boucles sécantes les unes par rapport aux autres. Une forme intérieure est alors décrite toute de luxuriance végétale, objectivée pour être présentée au regard, maîtrisée, pour ainsi dire domptée par la figure de l'auteur mise à part et rendue dans le manuscrit par saint Jérôme. Au feuillet $235 \mathrm{r}^{\circ}$, dans l'initiale $C$, au début du Prologue au Livre de Job, Jérôme est encore une fois assis en auteur enseignant et domptant le corps de la lettrine, les pieds sur l'escabeau à même le cou de l'animal (fig. 3). 
Fig. 3 - Bible de Saint-Bénigne de Dijon, op. cit., fol. $235 \mathrm{r}^{\circ}$, initiale $C$.

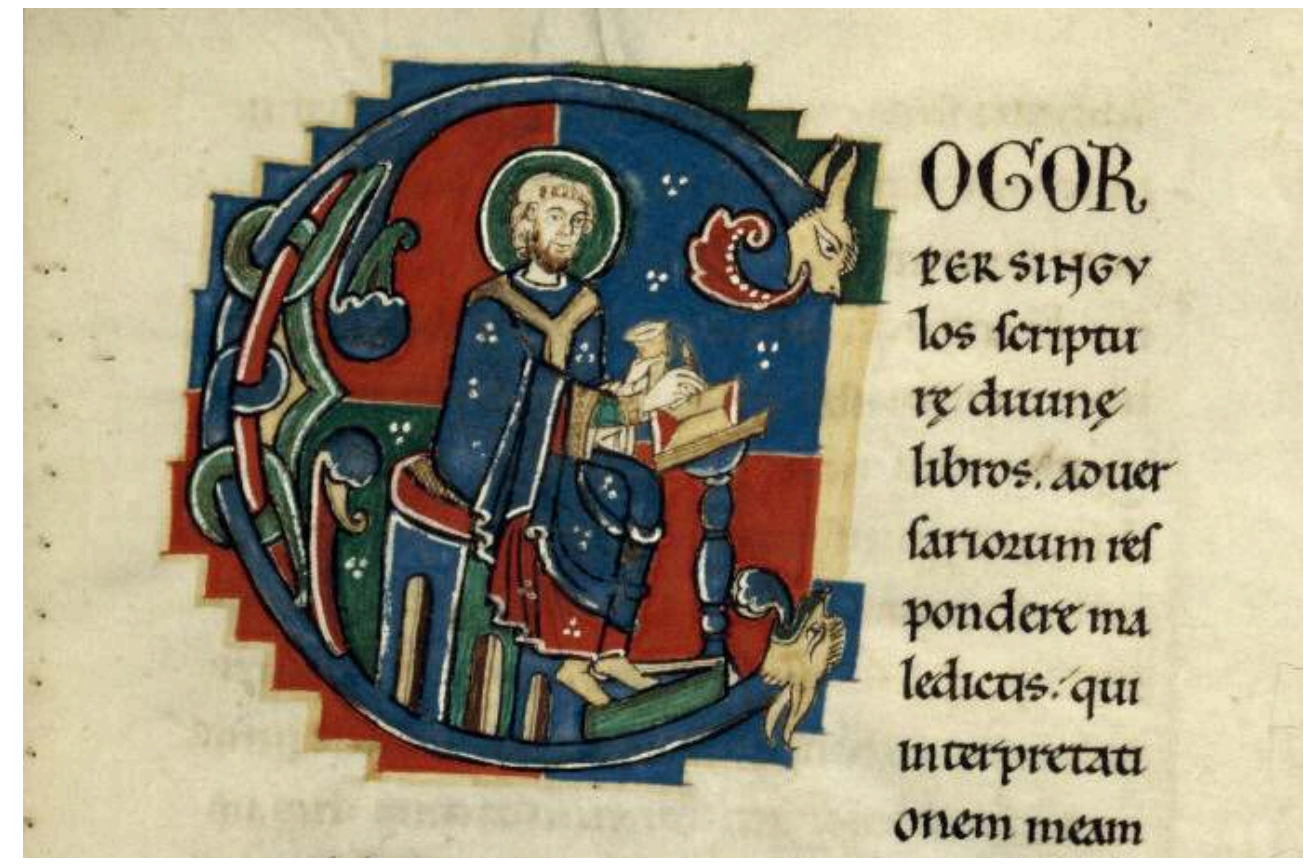

La lettre suscite un contexte d'une grande richesse puisqu'elle associe en elle-même, et entre ses différentes parties, les registres de l'animal et du végétal, en promouvant tout en haut de la hiérarchie, ainsi du même coup instituée, l'humain au cœur de l'animal et au centre de la composition, dominant toutes les autres parties, voire les transformant en les insérant aux autres figures de la présentation : l'auteur monastique est campé dans sa lettrine comme une autorité qui structure et ordonne, par rapport à elle, les formes environnantes ${ }^{67}$. Plus loin dans les feuillets, au verso du feuillet 288 , dans le Prologue composé par saint Jérôme au Livre de Salomon, l'auteur est présenté tout entier sur le fond peint à la feuille d'or, en lieu et place du végétal, et il s'est imposé comme l'exemplaire achevé de l'humain, c'est-à-dire du moine au sommet de la société des hommes, à l'exclusion des autres règnes, y compris du règne animal (fig. 4) ${ }^{68}$. 
Fig. 4 - Bible de Saint-Bénigne de Dijon, op. cit., fol. $288 \mathrm{v}^{\circ}$, initiale $C$.

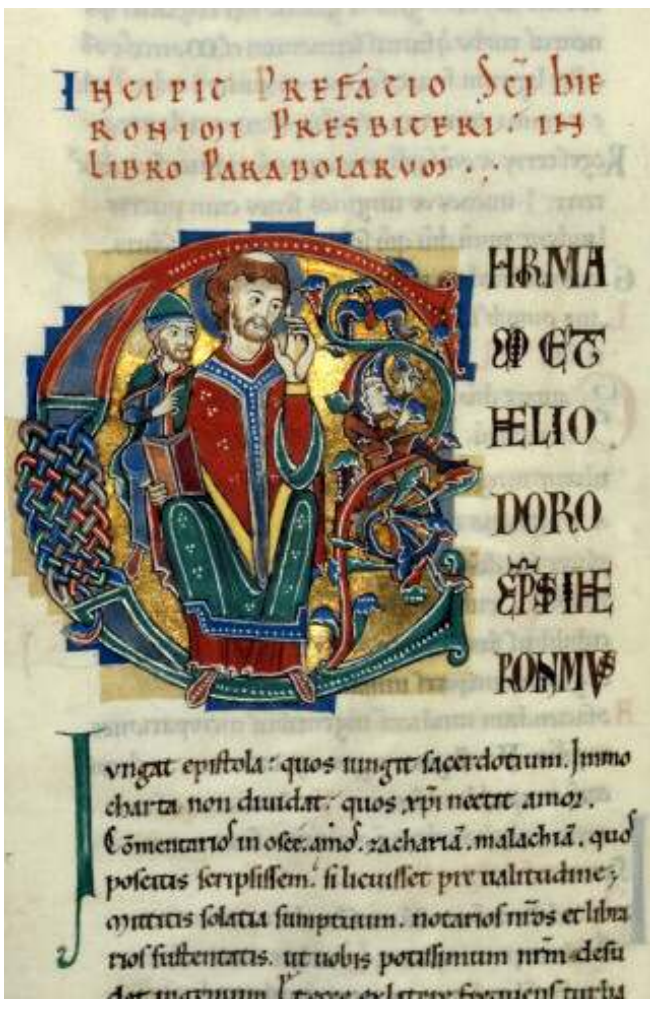

C'est du travail intérieur à la lettrine, donc du travail de l'artiste, qu'est suscitée la figure de l'auteur peu à peu saisie sur un fond de présentation, ce champ, qui, tout à la fois, l'inscrit dans un processus dynamique de création, le produit aux regards et lui confère sa pertinence par rapport à l'affirmation de l'auteur monastique, l'homme de l'Église et l'homme par excellence. Les traités et les différents autres textes contemporains rejoignent ces pratiques, ils ne les fondent absolument pas. Et le symbolisme architectural n'agit qu'après coup, tel un argument supplémentaire qui viendrait s'ajouter, pour mieux la rendre perceptible aux sens, à l'ecclésiologie qui est en train de s'écrire ${ }^{69}$. Les reformulations du $\mathrm{XII}^{\mathrm{e}}$ siècle sont aussi les marques d'une recomposition de toute la création universelle au cœur de laquelle est installé le moine.

À l'inverse de la perspective «internaliste » de Pierre Hadot, dans ces formulations iconographiques, le labeur, l'exercice du traducteur - qui est aussi un exercice de transformation -, la méditation, sont inscrits en articulation active avec ce qui se trouve dans le champ. Nous pourrions voir ici encore une expression de la triade structurelle exercitia, spiritualia, corporalia, la transformation/traduction se déclinant à l'intérieur d'un ensemble articulé intérieur/extérieur dans le monde, pas en dehors de lui. Mais essayer de faire correspondre les images aux mots dégagés des textes n'est plus une solution, si elle ne l'a jamais été. Les images pointent aussi vers un rapport qui dépasse celui du moine aux prises avec son intériorité et son extériorité, avec son esprit et son corps, c'est-à-dire avec tout ce qui s'étend au-delà de lui-même. Ainsi visualisées, les images pointent vers le réseau matériel des figures qui saturent le "fond de l'image » ou, mieux, vers le réseau dans lequel Jérôme est saisi, inscrit à son tour dans le champ de ce qui est montré par l'artiste en son travail. 


\section{Exercices d'auto-réflexivité. Les réflexes disciplinaires ${ }^{70}$} nous avons choisi de traiter, en historiens, de la notion des « exercices spirituels », qui doit retenir notre attention. Travailler sur une notion qu'on veut historiquement attester, inscrite dans la continuité du temps, mais dont les bases historiques ne sont pas effectivement démontrées, nous a causé d'emblée des difficultés. Devant une généralisation de prime abord inconfortable, le réflexe a été de l'inscrire dans le temps : comment l'expression répandue par le libellum d'Ignace de Loyola au milieu du $\mathrm{xVI}^{\mathrm{e}}$ siècle avait-elle été surinvestie au $\mathrm{xx}^{\mathrm{e}}$ siècle dans des contextes précis ? Le test par l'étude des mots et de l'iconographie a conduit à repérer un moment particulier, le nouveau monachisme du XII ${ }^{\mathrm{e}}$ siècle, puis a invalidé l'idée d'une permanence ou d'une « origine » tardo-antique et, par conséquent, a fait ressortir les limites mêmes de toute généralisation, en particulier de celle avancée par Pierre Hadot. Sur le fond, et pour différentes raisons, celui-ci défendait la philosophie face à la théologie comme "sagesse supérieure ", en traitant de l'histoire à très grands coups de pinceaux sans s'arrêter au noyau du XII ${ }^{e}$ siècle. Dès lors, et selon notre approche, nous avons voulu établir un autre ordre en mettant en avant précisément l'histoire. L'exercice de l'interdisciplinarité a toutefois renforcé la disciplinarité, ici en l'occurrence et par nos choix délibérés, l'histoire. Nous poserons donc la question de savoir à quel(s) niveau(x) de formulation et d'abstraction nous placer pour pratiquer une interdisciplinarité. Estil suffisant de postuler un objet commun - «l'homme ", "les choses ", "la société ", "les sociétés "-, tout aussi susceptible de généralisation? Un début de réponse pourrait consister à se défaire des paradigmes mous et à construire des concepts solides. Le " modèle ", ou encore le " modèle antique », bien que largement accepté à la suite d'un large consensus fondé, en apparence, sur le sens commun, ne résiste, par exemple, ni à un effort de contextualisation, ni à un effort de critique théorique. De même, les notions toutes prêtes du "portrait d'auteur " et de la «tradition", sans omettre celle bien facile du "symbolisme", ne résistent pas davantage à une analyse suivie des œuvres prises en compte, depuis les conditions de leur fabrication jusqu'au regard posé sur les savoir-faire et les maîtrises techniques dont elles résultent.

nous est apparu également que procéder par analogie - les philosophes antiques, les moines au Moyen Âge, les jésuites bien après, et d'autres acteurs ensuite - ne faisait que renforcer et justifier le jeu des projections contemporaines sur le passé. C'est à dessein alors que, pour les deux approches développées, nous n'avons pas pensé en termes de "relation» entre «texte et image », bien au contraire, et que nous avons donc essayé de pointer certains éléments du problème qui pourraient s'avérer structurels, ou structurants, et constituer une base plus solide pour une réflexion conceptuelle.

Ce sont là quelques jalons dans une recherche plus ample, en cours de construction.

Reçu : 4 octobre 2012 - Accepté : 4 novembre 2012 


\section{NOTES}

1. Nous remercions François Athané de nous avoir invités à intervenir lors de la journée d'études en hommage à Pierre Hadot, "Vivre la philosophie », organisée par l'Association des professeurs de philosophie de l'enseignement public (APPEP) et le Centre international d'études de la philosophie française contemporaine de l'École normale supérieure (CIEPFC), qui s'est tenue à Paris, le 16 juin 2012. Nous empruntons à l'article issu de cette intervention certains des développements de cet essai : E. MAGNANI et D. Russo, «Exercices spirituels, études de mots, expériences d'images : réflexions sur l'exemple du Moyen Âge ", L'enseignement philosophique, Revue de l'APPEP, 63/1 (2012), p. 48-61.

2. À titre d'exemple, nous renvoyons à D. RUsso, "Penser en interdisciplinarité dans une UMR de sciences humaines et sociales (2007-2011)», Bucema, 15 (2011), p. 305-318 [http:// cem.revues.org/index12112.html] et à E. MAGNANI (dir.), Don et sciences sociales. Théories et pratiques croisées, Dijon, 2007.

3. DIJon, Bibliothèque municipale, ms. 2. Notre étude porte, en particulier, sur les initiales figurées aux feuillets $6 \mathrm{r}^{\circ}[D], 100 \mathrm{r}^{\circ}[U], 235 \mathrm{r}^{\circ}[C]$ et $288 \mathrm{v}^{\circ}[C]$. Sur le manuscrit et sa datation autour du deuxième quart $\mathrm{du} \mathrm{XII}^{\mathrm{e}}$ siècle, y. ZALUSKA, L'enluminure et le Scriptorium de Cîteaux au XII siècle, Cîteaux, 1989, p. 144-147, notice 88, p. 262-263, et « Annexe II », p. 276-277, et Y. ZALUSKA, collab. M.-F. DAMONGEOT, F. SAULNIER et G. LANOË, Manuscrits enluminés de Dijon, Paris, 1991 (Corpus des manuscrits enluminés des collections publiques des départements, 1). Mise en situation dans l'histoire, C.M. MAlone, Saint-Bénigne de Dijon en l'an Mil. Totius Galliae Basilicis Mirabilior. Interprétation politique, liturgique et théologique, Turnhout, 2009 (Disciplina monastica, 5).

4. C'est par l'entremise de Michel Foucault (1926-1984), qui s'intéresse alors à la vie comme « réel de la philosophie ", que Pierre Hadot se présenta au Collège de France. Pierre Hadot est revenu sur leurs rapports divergents dans $\mathrm{P}$. HADOT, La philosophie comme manière de vivre. Entretiens avec J. Carlier et A. I. Davidson, Paris, 2001, p. 215-217. Pour les rapprochements possibles entre les deux pensées, voir, par exemple, T. FLYNN, «Philosophy as a way of life: Foucault and Hadot", Philosophy and social criticism, 31/5-6 (2005), p. 609-622 ; D. LORENZINI, « La vie comme "réel" de la philosophie. Cavell, Foucault, Hadot et les techniques de l'ordinaire », in S. LAUGIER (dir.), La voix et la vertu. Variétés du perfectionnisme moral. Éthique et philosophie morale, Paris, 2010, p. 469-487.

5. P. HADOT, La philosophie..., op. cit. Sur le parcours de P. Hadot, voir Ph. HOFFMANN, « Pierre Hadot (1922-2010) ", Annuaire EPHE, Sciences religieuses, 119 (20102011), p. XXXIII-XLII.

6. VICTORIN L'AFRICAIN, Traités théologiques sur la Trinité. 1-2, texte établi par P. HENRY, intro., trad. et notes par P. HADOT, Paris, 1960 (Sources chrétiennes, 68-69) ; Р. HADOT, Porphyre et Victorinus. Paris, 1968 ; ID., Marius Victorinus : recherches sur sa vie et ses œuvres, Paris, 1971.

7. P. HADOT, Plotin ou la simplicité du regard, Paris, $1963,1997^{2}$; Р. НADOT (dir.), Les écrits de Plotin [Ennéades], Paris, $1988,1991^{2}$ et $1994^{3}$.

8. AMBROISE DE MILAN, Apologie de David, intro., texte latin, notes et index par P. HADOT, trad. par M. CORDIER, Paris, 1977 (Sources chrétiennes, 239).

9. P. HADOT, La citadelle intérieure: introduction aux «Pensées» de Marc Aurèle, Paris, 1992 ; MARC AURÈLE, Écrits pour lui-même, texte et trad. par P. HADOT, avec la collab. de C. LUNA, Paris, 1998.

10. P. HADOT, Qu'est-ce que la philosophie antique?, Paris, 1995.

11. Р. НADOT, «Exercices spirituels", Annuaire de la Ve section de l'École pratique des hautes études, 84 (1975-1976), 1977, p. 25-70, repris dans ID., Exercices spirituels et philosophie antique, Paris, 1981 (1987², $1993^{3}$ ), recueil d'articles, 1, p.13-58. Nous utilisons, dans les citations ci-dessous, l'édition de 1987.

12. P. RABBOW, Seelenführung. Methodik der Exerzitien in der Antike, Munich, 1954. 
13. I. DE LOYOLA, Exercitia spiritualia. Auctore S. Ignatio de Loyola. Andreas Frusius latine vertit. Praemittuntur Annotationes quaedam aliquid adferentes intelligentiae ad Exercitia spiritualia, Rome, 1548 (voir Monumenta ignatiana. Series $2^{a}$ Exercitia spiritualia S. Ignatii de Loyola et eorum directoria. Nova editio, Rome, Institutum historicum Societatis Iesu, 1969).

14. Р. НADOT, « Exercices spirituels », op. cit., p. 14-15.

15. Р. HADOT, « Exercices spirituels », ibid., p. 15-16; ID., « Epistrophè et Metanoia dans l'histoire de la philosophie », in Actes du XI ${ }^{e}$ Congrès international de philosophie, Bruxelles, 1953, t. 12, p. 31-36.

16. P. HADOT, « Exercices spirituels », ibid., p. 16, p. 17.

17. P. HADOT, "Exercices spirituels ", ibid., p. 17 ; ID., " La physique comme exercice spirituel ou pessimisme et optimisme chez Marc-Aurèle ", Revue de théologie et de philosophie, 1972, p. 225-239 ; repris dans Exercices spirituels..., op. cit., p. 119-133. Pour une approche plus développée de ces questions, en particulier de la "nature ", ID., Le voile d'Isis. Essai sur l'histoire de l'idée de nature, Paris, 2004, en part. chap. 2, "De la Phusis à la nature» (p.35-45). Sur la vision et la problématique développée du «regard», ID., Plotin ou la simplicité du regard, Paris, 1963 ("La recherche de l'absolu»), 1997², en part. chap. 2 ("Niveaux du moi », p. 23-45), 4 ("Amour», p. 105-106) et 6 (« Douceur», p. 156-157). Selon une étude historique des niveaux du moi et de la "vision », pour une autre problématique du « regard », G. SIMON, Le regard, l'être et l'apparence dans l'optique de l'Antiquité, Paris, 1988 ; ID., Sciences et savoirs aux XVI et XVII siècles, Lille, 1996, sur les conséquences de tout ce mouvement d'idées pour la vision et pour la définition du regard par la modernité.

18. J. DOMAŃSKI, La philosophie, théorie ou manière de vivre? Les controverses de l'Antiquité à la Renaissance, préface de P. HADOT, Paris/Fribourg, 1996 (Vestigia. Pensée antique et médiévale, 18).

19. P. HADOT, Qu'est-ce que la philosophie antique?, op. cit., chap. $\mathrm{x}$ (« Le christianisme comme philosophie révélée », p. 355-378) et XI (« Disparitions et réapparitions de la conception antique de la philosophie », p. 379-407), ici p. 381-382. Voir aussi ID., La philosophie..., op. cit., p. 182-185.

20. Р. НАDOT, La philosophie..., ibid., p. 390-391 et 396.

21. P. RABBOW, Seelenführung..., op. cit.

22. Voir G. LUCK (1926-), «Review of Paul Rabbow, Seelenführung. Methodik der Exerzitien in der Antike ", in ID., Ancient Pathways and Hidden Pursuits : Religion, Morals, and Magic in the Ancient World, Ann Arbor, 2000, p. 35-38 [repris de Gnomon, 28 (1956), p. 268-271]; R. J. NEWMAN, "Cotidie meditare. Theory and Practice of the meditatio in Imperial Stoicism ", in W. HAASE et H. TEMPORINI (dir.), Aufstieg und Niedergang des römischen Welt. Geschichte und Kultur Roms im Spiegel der neueren Forschung, Berlin/New York, 1989, t. 2, bd. 36/3, p. 1473-1517, ici p. 1476-1477, n. 6.

23. I. HADOT, Seneca und die griechisch-römische Tradition der Seelenleitung, Berlin, 1969. Une liste des comptes rendus de cet ouvrage est donnée dans la bibliographie établie par F.-R. CHAUMARTIN, "Quarante ans de recherche sur les œuvres philosophiques de Sénèque (Bibliographie 1945-1985) », in W. HAASE et H. TEMPORINI (dir.), Aufstieg und Niedergang..., ibid., p. 1546-1605, ici p. 1555 .

24. P. HADOT, «Exercices spirituels antiques et "philosophie chrétienne" ", dans ID., Exercices spirituels..., op. cit., p. 59-74.

25. Il s'agit du titre du premier chapitre de ses entretiens, La philosophie..., op. cit., p. 17-60.

26. M. RUIZ JURADO, S. J., «El instituto de espiritualidad de la Gregoriana desde su nacimiento a nuestros días. Su aporte a la teología espiritual », Gregorianum, 90/2 (2009), p. 358-370.

27. M. RUIZ JURADO, «El instituto de espiritualidad de la Gregoriana... », ibid., p. 362-363 (sur L. Hertling).

28. L. HERTLING, S. J., " De usu nominis exercitiorum spiritualium ante S. P. Ignatium ", Archivum Historicum Societatis Iesu, 2 (1933), p. 316-318. L. Hertling, qui va enseigner à la Gregoriana à partir de 1936, avait publié auparavant Lehrbuch des aszetischen Theologie (Innsbruck, 1930) et Das geistliche Leben (Vienne, 1933), puis Theologia ascetica, cursus brevior (Rome, 1939 et 1944). Les 
auteurs qui avaient traité des origines des Exercices spirituels d'Ignace de Loyola, auparavant, n'avaient pas recherché, à notre connaissance, jusqu'au haut Moyen Âge.

29. «Exercices spirituels» par J.LECLERCQ (1911-1993), A. RAYEZ, P. DEBONGNIE et C. SCHMERBER, Dictionnaire de spiritualité. Ascétique et mystique. Doctrine et histoire, t. 4, Paris, 1961, col. 1902-1949. Ce dictionnaire est consultable en ligne [http://www.dictionnairedespiritualite.com]. L'entrée «exercices spirituels » ne figure pas dans des dictionnaires ecclésiastiques antérieurs; elle est absente, par exemple, du Dictionnaire de théologie catholique, A. VACANT et E. MANGENOT (dir.), Paris, 1902-1950, 15 tomes. Elle figure, cependant, dans les dictionnaires postérieurs, comme le Theologische Realenzyklopädie, G. MÜLLER, H. BALZ et G. KRAUSE (dir.), vol. 10, Berlin/New York, 1982, entrée «Exerzitien » par J. E. VERCRUYSSE, S. J., et M. SEITZ, p. 698-707 ou comme le Dizionario Enciclopedico di Spiritualità, t. 2, E. ANCILLI et Pontificio Istituto di Spiritualità del Teresianum (dir.), Rome, 1990, entrée « Esercizi spirituali », par I. IPARRAGUIRRE, S. J., p. 911-918; dans les deux cas, les articles se concentrent sur les Exercices d'Ignace de Loyola et leur postérité, en renvoyant, pour la rétrospective historique, à l'article, devenu référence, du Dictionnaire de spiritualité.

30. L. FEBVRE, La religion de François Rabelais. Le problème de l'incroyance au XVI siècle, Paris, 1947.

31. A. GRABAR, Les voies de la création en iconographie chrétienne. Antiquité, Moyen Âge, (Princeton, 1968), publ. en fr. Paris, $1979\left(1994^{2}\right)$, pour une synthèse de ses idées sur ces " continuités » de l'Antiquité vers le Moyen Âge qu'il exprimait dès la thèse sur L'Empereur dans l'art byzantin. Recherches sur l'art officiel de l'Empire d'Orient, Paris, 1936 (Publications de la Faculté des lettres de l'université de Strasbourg, 75). Voir le compte rendu de L. Marcel dans la Revue belge de philologie et d'histoire, 16/3-4 (1937), p.763-769. Contre la thèse des continuités et fondant tout son raisonnement sur les « ruptures » entre Antiquité gréco-romaine et Antiquité chrétienne, haut Moyen Âge, TH. F. MatThEws, The Clash of Gods. A Reinterpretation of Early Christian Art, Princeton, $1993\left(1995^{2}\right.$; 1999³) ; compte rendu et discussion par D. Russo, Revue de l'art, 106 (1994), p. 97-98. Pour une présentation de l'ensemble de l'œuvre de Grabar, M. G. MuzJ, Un maître pour l'art chrétien, André Grabar. Iconographie et Théologie, Paris, 2005.

32. En particulier, Ed. DE BRUYNE, Études d'esthétique médiévale, 3 vol., Bruges, 1946, t. 1, «Les fondateurs "; «La civilisation carolingienne». Voir R. HEYNICKX, «Le chantier de la tradition. Réflexions d'Edgar De Bruyne sur la culture moderne pendant l'entre-deux guerres : possibilités et limites du néo-thomisme ", Revue d'histoire ecclésiastique, 100/2 (2005), p. 519-545; pour une discussion, D. Russo, «Les lectures de l'art sacré en France et en Europe au tournant des années 1880-1920. Autour du "médiévalisme" ", in F. CHAUBET (dir.), Catholicisme et monde moderne aux XIX et $\mathrm{XX}^{e}$ siècles. Autour du "Modernisme", Dijon, 2008, p. 91-102.

33. Voir É. BenVEnISTE, Dernières leçons. Collège de France (1968-1969), éd. et prés. par J.-C.. CoQUET et I. FENOGLIO, préface par J. KRISTEVA, postface par T. TODOROV, Paris, 2012.

34. Voir les travaux pionniers, à leur époque : J. SEZNEC, La survivance des dieux antiques. La tradition mythologique et sa place dans l'humanisme et dans l'art de la Renaissance, Londres, 1939, 1940² ; trad. en angl. [USA] par B. SESSIONS, New York, 1954 ; publ. en France, Paris, 1980, 1994² ; J. AdHÉMAR, Influences antiques dans l'art $d u$ Moyen Âge français. Recherches sur les sources et les thèmes d'inspiration, Londres, 1937 ; publ. en France, Paris, 2000 (c'est nous qui soulignons).

35. P. COURCELlE, Connais-toi toi-même, Paris, 1975, entre autres ouvrages sur Virgile et sur saint Augustin. Pierre Courcelle, mais aussi Jean Seznec et Jean Adhémar, dépendent beaucoup de W. JAEGER (1888-1961), Paideia, (3 vol., Berlin, 1933-1947), trad. en fr. par A. et S. DeVYVER, Paris, $1964,1988^{2}$, qui faisait reposer l'éducation et les moyens de la transmission des savoirs (l'agora, le gymnase, le rouleau) sur une conception de l'universel : voir Livre 1, chap. 9, «La spéculation philosophique : la découverte de l'ordre universel ", en part. p. 186-226 de la trad. fr (c'est nous qui soulignons). 
36. G. AGAMBEN, De la très haute pauvreté. Règles et forme de vie. Homo sacer IV, 1, trad. fr. par J. GAYRAUD, Paris, 2011. Voir aussi E. CoccIA, «Regula et vita. Il diritto monastico e la regola francescana », Medioevo e Rinascimento, 20 (2006), p. 97-147.

37. F. MORETTI, «Conjectures on wolrd literature », New Left Review, 1 (2000), p. 54-68, ici p. 57, et ID., Graphes, cartes et arbres. Modèles abstraits pour une autre histoire de la littérature, Londres/New York, 2005, trad. fr. E. DOBENESQUE, Paris, 2008.

38. En particulier dans Mediae latinitatis lexicon minus, par J. F. NIERMEYER, Leiden, $1976^{1}$ (CD-ROM). Le mot français « exercice » est utilisé dans 21 entrées pour traduire des mots désignant, entre autres, l'exercice d'une fonction (bajulus; comes) ; d'un droit, d'un pouvoir, d'une activité (honor ; legatio; mansura...); la gestion, l'administration (administratio ; gestus); l'école ; l'étude, la formation, l'exercice intellectuel; l'exercice spirituel (gymnasium/gignasium - gr.). Le mot latin exercitium est traduit par maniement; travail; pratique morale, ascèse, exercice spirituel ; épreuve, tourment ; œuvre littéraire, exercice de rhétorique ; affaire, occupation.

39. http://www.intratext.com/IXT/LAT0001/_INDEX.HTM. La Vulgate contient 626928 mots. Exercitium et ses dérivés sont très peu usités dans la Vulgate : huit hapax (dans Genèse 34, 10 ; II Paralipomènes 19, 6; I Timothée 4, 8 ; Hébreux 12, 11 et 5, 14 ; II Pierre 2, 14 ; II Maccabées 15, 12 et 4, 14) et un seul mot récurrent dix fois, exercitus, dans son acception militaire et d'armée (Josué 10, 5 ; Judith 2, 8; 5, 27 et 7, 1; Psaumes 43, $10 ; 59,12$ et 107, 12 ; I Maccabées 10, 36 et 12, 43 ; Matthieu $22,7)$.

40. Patrologiae cursus completus omnium SS. Patrum, doctorum scriptorumque ecclesiasticorum sive Latinorum, sive Graecorum. Patrologia Latina, publ. par J. P. MIGNE (1800-1875) entre 1844 et 1855 ; 217 volumes.

41. Le logiciel libre PhiloLogic a été développé par Mark Olsen et son équipe à l'université de Chicago [http://philologic.uchicago.edu/]. Nous remercions Alain Guerreau et Nicolas Perreux pour leur aide à l'installation. Sur l'analyse sémantique appliquée aux textes médiévaux voir, notamment, A. GUERREAU, L'avenir d'un passé incertain. Quelle histoire du Moyen Âge au XXI siècle?, Paris, 2001, p. 191-237, et ID., «Le champ sémantique de l'espace dans la Vita de saint Maïeul (Cluny, début du XI ${ }^{\mathrm{e}}$ siècle) », Journal des savants, 1997, p. 363-419.

42. Voici les 16 formes retrouvées, suivies de leur fréquence: exerciti (13), exercitia (519), exercitiaque (1), exercitibus (396), exercitibusque (5), exercitii (204), exercitiis (409), exercitio (837), exercitione (1), exercitionem (1), exercitior (2), exercitiorum (36), exercitis (3), exercitium (860), exercitiumque (3), exercitius (1).

43. Matth. 22, $7:$ Rex autem cum audisset iratus est et missis exercitibus suis perdidit homicidas illos et civitatem illorum succendit (« Le roi se mit en colère ; il envoya ses troupes, fit périr ces assassins et incendia leur ville »).

44. K. ALLEN SMITH, War and the Making of Medieval Monastic Culture, Woodbridge, 2011.

45. I Tim. 4, 7-8: Exerce autem teipsum ad pietatem. Nam corporalis exercitatio ad modicum utilis est, pietas autem ad omnia utilis est (« Exerce-toi plutôt à la piété. L'exercice corporel, en effet, est utile à peu de chose, tandis que la piété, elle, est utile à tout »).

46. « Exercices spirituels ", Dictionnaire de spiritualité, op. cit., col. 1904.

47. De praedestinatione et gratia, éd. PL 45, col. 1671 (texte pseudo-augustinien).

48. Regularis concordia monachorum Anglicae nationis (éd. PL 137, col.490C). Voir The monastic agreement of the monks and nuns of the English nation drawn up at the Synod of Winchester by St. Dunstan, St. Ethelwold, and others, éd. trad. Dom Th. Symons, s. 1., 1953.

49. In festo S. Andreae apostoli; Sermones de diversis; Sententiae (éd. PL 183, col. 512A, 638C et 749C). Voir Sancti Bernardi Opera, éd. J. LECLERCQ et H. M. ROCHAIS, Rome, 1957-1977 (Sermones in natali sancti Andreae, 1968, t. 5, p. 427-440, sermo 2, p. 438 ; Sermo de altitudine et bassitudine cordis, 1968, t. 5, p. 214-216, p. 215 ; Sententiae, 1972, t. 6, 2, p. 7-255, sententia 7, p. 9). 
50. Sermones de tempore ; Speculum charitatis ; Compendium speculi charitatis (éd. PL 195, col. 360C, 535B, 608A et 626B). Voir AeLREdus RIEUALLENSIS, Sermones I - LXXXIV, éd. G. RACITI, Corpus christianorum. Continuatio medievalis, 2A, Turnhout, 1989, Sermo 21 ; ID., De speculo caritatis, éd. C.H. TALBOT, Corpus christianorum. Continuatio medievalis, 1, Turnhout, 1971, p. 5-161, lib. 1, cap. 31 ; lib. 3, cap. 34 ; ID., Compendium speculi caritatis, ibid., p. 175-238, cap. 7.

51. Octo puncta perfectionis assequendae (éd. PL 184, col.1181D et 1186A). Voir M. DELCogliano, "Cistercian Monasticism in the Silver Age: Two Texts on Pratical Advice ", Cistercian Studies Quaterly, 45/4 (2010), p.421-452 qui considère ce texte comme étant l'œuvre d'un auteur cistercien anonyme, datant de la fin du XII ${ }^{\mathrm{e}}$ ou du début du XIII ${ }^{\mathrm{e}}$ siècle.

52. Epistolae, 254 (éd. PL 153, col. 893D). Voir Lettres des premiers chartreux: (II) les moines de Portes, Bernard, Jean, Étienne, texte critique, trad. et notes par " un chartreux », Paris, 1980 (Sources chrétiennes, 274), p. 56.

53. De differentia sacrificii Abrahae a sacrificio B. Mariae Virginis ; De eruditione hominis interioris (éd. PL 196, col. 1048D, 1242D, 1249C et 1304B).

54. Expositio altera super Cantica canticorum (éd. PL 180, col. 546C) ; Epistola, 310 (éd. PL 184, col. 322B et 326A). Voir GUILLAUME DE SAINT-THIERRY, Exposé sur le Cantique des cantiques, texte latin, intro. et notes J. M. DECHANET, trad. fr. M. DUMONTIER, Paris, 1962 (Sources chrétiennes, 82), p. 400 ; ID., Un traité de la vie solitaire. Lettre aux frères du Mont-Dieu (Epistola ad Fratres de Monte Dei), into., trad. et notes M. M. DAVY, Paris, 1946 (Études de philosophie médiévale, 29), p. 175 et $\S 42$ et 57.

55. De confessione sacramentali (éd. PL 207, col. 1087D).

56. Opusculum in verba : Ad quid venisti? (éd. PL 184, col. 1191A); Vitis mystica (éd. PL 184, col. 653D et 659C) [textes pseudo-bernardins].

57. Lexicogramme réalisé avec le logiciel TreeCloud.org [http://www2.lirmm.fr].

58. J. WIRTH, L'image à l'époque romane, Paris, 1999, en part. chap. 3, «La restructuration de l'image ", "L'image et le champ ", (p. 183-191), en citant Adam le Prémontré et son traité De triplici Tabernaculo (1180-1181), pars. 2, éd. PL 198, col. 683-744, mais en le projetant ensuite dans une construction de symbolisme architectural qui renverrait, plus ou moins, au fondement christique de la composition.

59. Nous pensons, en particulier, à G. AGAMBEN, Le règne et la gloire : pour une généalogie théologique de l'économie et du gouvernement. Homo sacer, II, 2 (trad. fr. J. GAYRAUD et M. RUEFF, Paris, 2008), qui revient sur les symboles des cérémoniaux du pouvoir, du trône, de la couronne, de la pourpre, des faisceaux romains, et qui s'interroge sur le problème de la généalogie inédite des formes et de la fonction du consensus, et de ses supports, dans des sociétés à différents moments de leur histoire. Nous renvoyons également à ID., Opus Dei, Archéologie de l'office, Homo sacer, $V$ (trad. fr. M. RUEFF, Paris, 2012), qui scrute les racines du nouveau paradigme ontologique inventé par l'Église, à partir des phases successives de réforme, pour offrir à l'action humaine un pôle d'attraction étendu et constant, fait sans précédent dont la portée fut considérable sur la culture séculaire de l'Occident.

60. Р. НADOT, « Exercices spirituels », op. cit.

61. J.-P. SARTRE (1905-1980), L'imaginaire. Psychologie phénoménologique de l'imagination, Paris, 1940 ; $1948^{2}$; réimpr. 1980. Sur ces parallèles, Р. НADOT, La philosophie..., op. cit., en part. chap. 6 ( « Le discours philosophique comme exercice spirituel », p. 144-158), 7 (« La philosophie comme vie et comme quête de la sagesse ", p. 159-193) et 8 («De Socrate à Foucault. Une longue tradition », p. 194-228).

62. Première enquête d'envergure sur ces « séries de faits ", le travail d'E. DE BRUYNE (1898-1959), Études d'esthétique médiévale..., op. cit., en part. t. 1 (Les fondateurs. La civilisation carolingienne). Sur l'arrière-plan du travail de DE BRUYNE, la période de crise des années 1900-1920, R. HEYNICKX, «Le chantier de la tradition... », op. cit. Sur la reprise d'un prétendu fonds carolingien de «séries de signes », visuels et textuels, et sur la vie de ces «traditions » dans la longue durée, voir A. GRABAR, 
Les voies de la création en iconographie..., op. cit. Bien sûr, un cadre général idéaliste a été tracé par E. PANOFSKY (1892-1968), La perspective comme forme symbolique et autres essais, trad. fr. des essais écrits de 1915 à 1932 sous la dir. de G. Ballange, Paris, 1975; discussion de la conception panofskyenne de la renaissance de l'antique, et surtout du classique, dans l'art médiéval, et situation dans sa vision « humaniste » large, culturelle, par C. LANDAUER, «Erwin Panofsky and the Renascence of the Renaissance », Renaissance Quarterly, 47/2 (1994), p. 255-281.

63. Discussion du mot "champ», introduit jadis par Meyer Schapiro (1904-1996), et de son emploi dans l'analyse de la peinture ou de l'enluminure, antique comme médiévale, par J.C. BAILly, Le champ mimétique, Paris, 2005, en part. « Définitions du champ » (p. 13-64).

64. Voir V. GAZEAU et M. GOULLET, Guillaume de Volpiano. Un réformateur en son temps (962-1031), Vita domini Wilhelmi, de Raoul Glaber, Caen, 2008 (compte rendu dans Bucema, 9 (2009) [http:// cem.revues.org/index11151.html]). C. M. MALONE, Saint-Bénigne de Dijon..., op. cit., en part. chap. 3 (Réforme et Renovatio, p.94-113) sur la réforme à Saint-Bénigne autour de l'an Mil. Sur les mécanismes en jeu et les réactualisations en séries qui s'opérèrent alors comme autant de réarticulations, voir toujours G. DUBY (1919-1996), L'an Mil, Paris, 1974, 1985², en part. chap. 3 (« Le visible et l'invisible », p. 63-102), 7 (« Nouvelle Alliance », p. 181-202) et 8 (« L'essor », p. 203-226). Pour la discussion autour du scriptorium de Cîteaux, Y. ZALUSKA, L'enluminure et le scriptorium de Cîteaux..., op. cit., en part. p. 144-147 et Annexe II, p. 276-277.

65. Bible de Saint-Bénigne de Dijon: DIjon, Bibliothèque municipale, ms. 2, fol. $6 \mathrm{r}^{\circ}$, initiale $D$, début du Prologue de saint Jérôme au Pentateuque. Le texte inscrit est: Desiderii mei desideratas accepi epistulas, qui quodam praesagio futurorum cum Danihele sortibus est nomen, obsecrantis ut translatum in latinam linguam de hebraeo sermone Pentateuchum nostrorum auribus traderem (...).

66. Ibidem, fol. $100 r^{\circ}$, initiale $U$, début de la Préface au Livre des Rois par saint Jérôme. Le texte qui se développe est: Viginti et duas esse literas apud Hebraeos, Syrorum quoque et Chaldeorum lingua testatur, quae hebraeae magna ex parte confinis est ; nam et ipsi viginti duo elementa habent eodem sono, sed diversis caracteribus (...).

67. Ibidem, fol. $235 \mathrm{r}^{\circ}$, initiale $C$, début du Prologue par saint Jérôme, supportant le texte : Cogor per singulos Scripturae divinae libros adversariorum respondere maledictis/ qui interpretationem meam (...).

68. Ibidem, fol. $288 \mathrm{v}^{\circ}$, initiale $C$, début du Prologue au Livre de Salomon, et texte inscrit : Cromatio et Heliodoro episcopis Hieronymus (...).

69. Selon une approche d'histoire de «la scène» et de sa progressive distinction des architectures chrétiennes, telle celle du Tabernacle, St. Lojkine réintroduit le symbolisme des formes et des figures de la pensée par le biais de la métaphore architecturale, dans «De l'allégorie à la scène : la Vierge-Tabernacle ", in B. PEREZ-JEAN et P. EICHEL-LojKINE (dir.), L'allégorie de l'Antiquité à la Renaissance, Paris, 2004, p. 509-531. Pour une remise en situation historique, à partir de l'examen d'un groupe de textes $\mathrm{du} \mathrm{XII}^{\mathrm{e}}$ siècle, au moment charnière de la construction d'une ecclésiologie redynamisée par la réforme de l'Église, voir D. IOGNA-PRAT, «Édification personnelle et construction ecclésiale ", in B. M. BEDOs-REZAK et D. IOGNA-PRAT (dir.), L'individu au Moyen Âge. Individuation et individualisation avant la modernité, Paris, 2005, p. 247-269 et 350-359, en part. p. 252-258 («La "fabrique" du Tabernacle »).

70. Sur le balancement entre l'interdisciplinaire et le disciplinaire, voir les travaux réunis par G. ORIGGI et F. DARBELLAY, Repenser l'interdisciplinarité, Genève, 2010. 
INDEX

Mots-clés : interdisciplinarité, Pierre Hadot, exercices spirituels

\section{AUTEURS}

ELIANA MAGNANI

CNRS/Artehis-UMR 6298

DANIEL RUSSO

Université de Bourgogne/Artehis-UMR 6298/IUF 